\title{
$\mathrm{N}$ a c h t r a g. ${ }^{1)}$
}

Während des Druckes des Speciellen Theiles sind mir folgende, 1884 und 1885 erschienene Abhandlungen, die auf die vorliegende Materie Bezug haben, zugekommen:

Blasius, W. Über Vogelbrustbeine. Journ. f. Ornithologie. XXII. Leipzig 1884. p. 228 f. (Kurzes Referat über einige besondere Bildungen an verschiedenen Vogelsterna, namentlich bei Limicolae, Psittaci, Cuculidae und Dendrochelidon).

Cunningham, D. J. The M. sternalis. Journ. of Anat. and Phys. XVIII. London p. 208 f.

Dollo, L. Première note sur le Simoedosaurien d'Erquelinnes. Bull. du Musée royal d'hist. nat. de Belgique. III. Brnxelles 1884. p. 172. („Foramen supracoracoïdien“ der Reptilien).

Helm, Fr. Über die Hautmuskeln der Vögel, ihre Beziehungen zu den Federfluren und ihre Functionen. Journ. f. Ornithologie. XXXII. Leipzig 1884. p. 321 f. (Umfangreiche und sehr fleissige Untersuchung über die zur Haut gehenden Theile der Mm. cucullaris, pectoralis, latissimus dorsi etc. etc. ["Hautmuskeln"] bei Colymbus glacialis, Anas clangula, Harelda glacialis, Mergus merganser, Anser cinereus, Anser domesticus, Cereopsis Novae Hollandiae, Chauna chavaria, Scolopax gallinago, Tringa minuta [?], Crex pratensis, Nothura maculosa, Gallus dornesticus, Phasianus colchicus, Goura coronata, Columba domestica, C. risoria, Buteo vulgaris, Asio brachyotus, Strix [Rhinostrix] mexicana, Athene cunicularia, Cypselus apus, Dendrocopus major, Gecinus viridis, Alauda arvensis, Corvus cornix, C. corone, Pica caudata, Sturnus vulgaris, Passer domesticus, Fringilla cannabina, Fr. coelebs, Loxia curvirustris, Parus coeruleus, Muscicapa grisola, 'Turdus merula, T. musicus. Bei der Untersuchung sind vornehmlich die Verbindungen mit der Haut und die Beziehungen zu den Federfluren und Rainen berïcksichtigt. Die Praeparation wurde in der Regel von innen her an der abgezogenen Haut geübt, wodurch mehr als in meinen Untersuchungen der Nachdruck auf die Verbindungen mit der Haut nebst Federfluren und Rainen gelegt werden konnte. Von der Litteratur ist auf das Hauptsächlichste Bezug genommen. Die Innervation wurde nicht berücksichtigt, die Bezeichnung der Muskeln meist im Anschlusse an die älteren Deutungen gegeben).

Shufeldt, R. W. Observations upon the Osteology of Podasocys montanus. Journ. of Anat. and Phys. XVIII. p. 86. London and Cambridge 1884. (Mit detaillirter Beschreibung des Skeletes).

Shufeldt, R. W. On the Osteology of Ceryle alpina. Journ. of Anat. and Phys. XVIII. p. 279 f.

Shufeldt, R. W. On the Osteology of Numenius longirostris, with Notes upon the Skeletons of other American Limicolae. Journ. of Anat. and Phys. XIX. p. 51 f. October 1884. (Mit vielen Specialangaben).

Sutton, J. B1. On the Nature of Ligaments II. Journ. of Anat. and Phys. XIX. p. 27 ff. October 1884. (Mit kurzer Notiz über das Verhalten der Endsehne des M. supracoracoideus [M. subclavius Surton] bei Columba. Die frühere Litteratur ist grösstentheils ignorirt).

Amans, P. C. Comparaisons des organes du vol dans la série animale. Ann. des scienc. nat. (6. ser.). XIX. Art. 2. Paris 1885. (Dus Kapitel über den Flugapparat der Vögel, p. 198 ff., enthält eine ziemlich genaue Beschreibung des Skeletes und der Flügelgelenke nebst ihren Bewegungen; die hauptsächlichsten Muskeln sind kurz angeführt).

Baur, G. Dinosaurier und Vögel. Eine Erwiderung an Herrn Prof. W. DaMes. Morph. Jahrb. X. Leipzig 1885. p. 446 f. (U. A. mit Notiz über die Clavicula bei den Ratiten).

Baur, G. "Note on the Sternal apparatus in Iguanodon". Zool. Anzeiger 1885. No.205. p. $561 \mathrm{f}$.

Beddard, F. E. A. Contribution to the Anatomy of Scopus umbretta. Proc. Zool. Soc. of London for 1884 (April 1885). p. 543. (Enthält u. A. eine gute Beschreibung der hauptsächlichsten Muskeln der vorderen Extremität etc. etc.).

1) Die seit 1884 erschienenen Lieferungen der grossen Werke von A. B. MEYeR (Abbildungen von Vogelskeleten) und A. Milne-Euwards et A. Grandidier (Oiseaux de Madagascar) standen mir leider nicht zu Gebote. 
Blix, M. Beobachtungen und Bemerkungen über den Flug der Vögel. Zeitschr. für Biologie. München und Leipzig 1885. p. 161 ff. (Mit zahlreichen Angaben über das Gewicht der Flugmuskeln, wobei die $\mathrm{Mm}$. pectoralis thoracicus, supracoracoideus und coraco-brachialis posterior zusammen gewogen wurden).

Dames, W. Entgegnnng an Herrn Dr. BAUr. Morph. Jahrb. X. Leipzig 1885. p. 603 f. (U. A. mit kurzen Bemerkungen über Furcula und Clavicula).

Dollo, L. L'Appareil sternal de l'Iguanodon. Revue des Questions scientifiques. October 1885. Bruxelles. p. 664 f. (Sehr eingehender und ausführlich begründeter Nachweis der sternalen Natur der von DoLlo bereits früher als Brustbeingebilde gedeuteten Skelettheile).

Filhol, H. Observations anatomiques, relatives à diverses espèces de Manchots. Recueil des Mém. Rapp. et Docum. relat. à l'observation du Passage de Vénus sur le soleil. III. 2. p. 65 ff. Paris 1885. (Enthält u. A. eine in der Hauptsache recht genaue und z. Th. vergleichende Beschreibung des Skeletes und der Muskulatur von Spheniscus demersus, Megadyptes antipodes, Eudyptes chrysolopha, Aptenodytes Pennantii, Dasyramphus Adeliae, Eudyptula minor, Pygosceles antarcticus mit prachtroll ausgeführten Abbildungen, sowie Angaben üiber den Plexus brachiajis).

Fürbringer, M. Über das Schulter- und Ellenbogengelenk bei Vögeln und Reptilien. Morph. Jahrb. XI. Leipzig 1885. p. 118 f.

Fürbringer, M. Über Deutung und Nomenklatur der Muskulatur des Vogelfügels. A. a. O.p. 121 f.

Fürbringer, M. Über die Nervenkanäle im Humerus der Amnioten. Morph. Jahrb. XI. p. 484. (Enthält auch Notizen über das Homologon des Canalis radialis bei den Vögeln).

Gadow, H. On the Anatomical Differences in the three species of Rhea. Proc. Zool. Soc. of London 1885. p. 308 f. (Mit sehr bemerkenswerthen Angaben über Umbildungen der Wirbelsäule, über die. Extremitäten etc. etc.).

Gadow, H. Brovs's Klassen und Ordnungen des Thierreiches. VI. 4. Vögel. Lief. 11 und 12. Leipzig und Heidelberg 1885. (Umfassende und sehr gute Darstellung der Muskulatur nach eigenen Untersuchungen nnd unter eingehender Benutzung vieler früherer Arbeiten. In den Deutungen folgt der Verfasser - von einigen wenigen, wohl durch die kurze Fassung meiner Mittheilung bedingten, Missverständnissen abgesehen - fast durchweg der von mir gegebenen Nomenclatur; eine genaue Unterscheidung. der von GADOw und von den früheren Autoren untersuchten Arten ist bei der zusammenfassenden Art seiner Darstellung nicht gut ausführbar. Von den mitgetheilten Befunden konnten im Folgenden nur die bemerkenswertheren und auffallenderen, sowie die von meinen Ergebnissen abweichenden mitgetheilt werden).

Hulke, J. W. Note on the Sternal Apparatus in Iguanodon. Quart. Journ. of Geolog. Soc. 1. VIII. 1885. XLI. London. p. $473 \mathrm{f}$.

Lindsay, Beatrice. On the Avian Sternum. Proc. Zool. So:. 1885. p. 684 f. (Bedentsame unter GADow's Leitung ausgeführte Untersuchung über die Entwickelung des Sternum und Brustgürtels von Struthio und mehreren Carinaten, nebst vergleichenden Ausführungen).

Strasser, H. Über den Flug der Vögel. Freiburg i/B. 1884 (Kürzere Zusammenstellung). — Über den Flug der Vögel. Jenaische Zeitschrift für Naturwissensch. XIX. Jena 1885. p. 174 ff. (Sehr umfangreiche und für die Theorie des Fluges hoch bedeutsame Abhandlung von bleibendem Werthe, die aber das hier behandelte Gebiet nur kurz streift ${ }^{1}$ ).

1) Nur auf einen Punkt sei in Kürze aufmerksam gemacht. STRAsser bemerkt hier (wie schon früher in der Luftsäcken der Vögel 1877) zu wiederholten Malen, dass die grösseren Vögel im Vergleiche mit den kleineren geringer entwickelte Flugmuskeln (namentlich Hebemuskeln der Flügel) besitzen, und hebt ferner (im Einklange mit Heцmнотт, 1873) hervor, dass das ansehnlichere Körpervolumen den grossen Vögeln mit Rücksicht auf den Aufenthalt in der Luft verbiete, ein betrïchtlicheres Gewicht von Flugmuskulatur mit sich zu führen. Damit verbessert ex zugleich seine frühere Angabe (1877), dass die grossen Vögel für die gleichen Leistungen eine geringere Masse von Muskulatur brauchten. - Ich habe im vorausgehenden Speciellen Theile an zahlreichen Stellen des osteologischen und myologischen Abschnittes den eingehenden Nachweis dieser quantitativen Differenz in der Ausbildung der Muskulatur bei grossen und kleinen Vögeln gegeben und zugleich wiederholt betont, dass für den mehr $r$ uhigen und scnwebenden Flug der grossen Flieger ein geringeres Quantum von Muskulatur genüge, da bei ihnen der passive Bewegungsapparat (Pneumaticität, Spannung der Furcula, Convexität des Sternum, Form und speciellere Structur der Flügel etc. etc.) höher a usgebildet sei. Dies harmonirt wohl in ler Hauptsache mit Strasser's 'neueren Ausführungen über diesen Punkt, wenn ich auch die Insufficienz der grossen Vögel für schnelle und energische Flügelbewegungen nicht besonders urgirt habe. 
Sutton, J. B1. The Nature of Ligaments. IV. Journ. of Anat. and Phys. XX. p. 39 ff. October 1885. London. (Notiz über die fibröse Schlinge für die Mm. Iatissimi dorsi von Spheniscus demersus und über den Expansor secundariorum, letztere im Anschlusse an die Garrop'schen Mittheilungen).

Vetter, B. Über die Verwandschaftsbeziehungen zwischen Dinosauriern und Vögeln. Festsschrift der naturwiss. Gesellsch. Isis in Dresden. Mai 1885. p. 109 f. (Bedeutungsvolle kritische Zusammenfassung über den jetzigen Stand der bezüglichen Frage, die auch kürzere specielle Mittheilungen über die Skeletverhältnisse der Odontornithes, der Archaeopteryx und der Dinosaurier enthält).

Wiedersheim, R. Uber die Vorfahren der heutigen Vögel. Humboldt IV. 6. Separatabdruck 1885. (Treffliche zusammenfassende Darstellung des Skeletbaues von Archaeopteryx und den Odontornithes Amerikas, namentlich auf Grund der Untersuchungen von DAMES und MARSH, mit Schlussbetrachtungen die Abstammung der heutigen Vögel betreffend).

Ausserdem war ich in der Lage, nachträglich noch folgende, bereits frïher erschienene Werke einzusehen, welche mir vorher nicht zugänglich waren:

Schmidt, M. Die Skelete der Hausvögel. Frankfurt a/M. 1867. (Mit trefflichen and geometrisch genauen Abbildungen der Skelete von Anas boschas, Anser cinereus, Gallus domesticus, Meleagris gallopavo und Columba livia var. domestica. Einige Ergebnisse nach Messungen an diesen Abbildungen wurden von mir in die vorhergehenden Tabellen eingetragen).

Owen, R. Memoirs on the Extinct Wingless Birds of New-Zealand, with an Appendix on those of England, Australia, New-Foundland, Mauritius and Rodriguez. London 1879. (Hauptwerk über die betreffende Frage. Zusammenstellung aller bezäglichen Abhandlungen des Verfassers über diesen Gegenstand mit zahlreichen Zusätzen und weiteren Beiträgen. Einige Zahlenangaben wurden den vorhergehenden Tabellen nach Messungen an 0 wex's Abbildungen zugefügt).

Aeby, Chr. Über das leitende Princip bei der Differenzirung der Gelenke. Beiträge zur Anatomie und Embryologie. Festschrift f. J. HenLE. Bonn 1882. (Mit kurzen, aber bedeutsamen Angaben über das Schulter- und Ellenbogengelenk der Reptilien und Vögel, welche bereits einen Theil meiner bezüglichen Befunde enthalten. Die frühere Litteratur über diesen Gegenstand ist mit wenigen Ausnahmen nicht berücksichtigt).

Der bemerkenswerthere Inhalt dieser Schriften ergiebt folgende Zusätze ${ }^{1}$ ):

\section{Osteologischer Abschnitt.}

A. Primärer Brustgürtel. A d p. 27. 28. Bei einem 4tägigen Embryo von Struthio sind Coracoid und Scapula noch nicht vereinigt (LinDs.aY).

1. Coracoideum. A d p. 35 f. Coracoid und Procoracoid sind bei dem 4tägigen Embryo von Struthio noch getrennt. Ebenso legt sich hier die Fenestra coracoidea von Anfang an und entsteht nicht (wie HoFrmaNN angiebt) erst secundär (LINDSAY). [Dies kommt ganz überein mit den von PARKER gemachten Angaben und stimmt mit der von mir geäusserten Auffassung]. Der 5tägige Embryo von Larus zeigt ein Coracoid, das mit dem von Struthio in der Sonderung von Coracoid s. str. und Procoracoid grosse Ähnlichkeit besitzt; doch fehlt ein eigentliches Foramen zwischen beiden. Später wachsen beide Theile zusammen, so dass das entwickelte Coracoid von Larus. Coracoid und Procoracoid in sich enthält (Lindsay). [Meine nachträglichen Untersuchungen an jungen Embryonen. von Larus canus konnten diesen wichtigen und auffallenden Befund leider nicht bestätigen; doch bin ich fern davon, daraufhin die Reellität der Angaben Lindsay's zu bezweifeln]. Im Ganzen tritt das Procoracoid bei Gallus. successive in Rückbildung (LINDSAY).

A d p. 44 f. Das Acrocoracoid (Tête du coracoidien) ist bei allen untersuchten Impennes stark nach vorn und. innen gekrümmt (FILHOL).

Ad p. 52 f. Der Proc. lateralis des Coracoid (Costal process. Shurcudr) zeigt sich bei Podasocys besonders ansehnlich und stark proximalwärts gekrümmt (SHUFELDT) [cf. auch Tabelle IX]. Bei den meisten Limicolae ist er schlank, bei Limosa uropygialis breit und quadratisch (SHuFELdT).

Ad p. 54 f. Dollo schlägt für das Foramen supracoracoideum der Reptilien diese Bezeichnung (For: supracoracoïdien) vor [was sich ganz mit der p. 54 von mir gegebenen Nomenclatur deckt]. Frunou findet diese Öffnung- 
(Trou sus-claviculaire FrLHol) bei den meisten Impennes oval; bei Aptenodytes Pennantii und Dasyramphus wird sie durch eine Incisur vertreten [cf. auch Tabelle X].

2. Scapula. A d p. 55. Im Anschluss an Owen und Dames etc. etc. erblickt Wiedersherm in der Seapula von Archaeopteryx eine echte Vogelscapula.

A d p. 57 f. Die grosse Breite der Scapula der Impennes wird gebührend hervorgehoben (FinHol).

Ad p. 65. 68. Der dorsale und ventrale Rand der. Scapula der Impennes zeigt ein sehr wechselndes Verhalten (FILHOL, s. die Ablandlung selbst).

Fossa glenoidalis humeri. A d $\dot{p}$. 68. ff. AxBy findet die coraco-scapulare Gelenkgrube für das Schultergelenk aus einer cylindrischen und kugelförmigen Fläche zusammengesetzt, im Ganzen sattelförmig. Dieselbe ist zugleich von einem mächtigen elastischen Faserpolster bekleidet. [Dies deckt sich in der Hauptsache mit einigen meiner bezüglichen Angaben, die damit ihre Priorität verlieren].

B. Secundärer Brustgürtel. 3. Clavicula. A d p. 75. 76. Die Claviculae sind bei Embryonen von Uria. sehr früh, früher als die sternalen Hälften vereinigt, weshalb der M. rectus abclominis in diesen Anfangsstadien noch mit der Clavicula verbunden ist und erst später durch die Vereinigung des Brustbeines aus dieser Verbindung gelöst wird. Bei Gallus existirt diese Beziehung des Muskels zur Clavicula nicht. In frühen Stadien ist die Clavicula von Gallus vorn mit dem Coracoid verbunden (Lindsay).

A d p. 84. 85. Die Spannung der. Furcula ist bei den Impennes recht verschieden ausgebildet; bei Eudyptes :z. B. ist sie ziemlich gering, bei Sphenișcus dagegen hoch entwickelt. Übrigens existiren hier vielfache Variirungen (FILног).

C. Primäres Brustbein. Sternum. Ad p. 95. Anm. 2. Vetter schreibt Archaeopteryx ein zweifellos carinates Sternum von nicht erheblichen Dimensionen zu. WIEDERsherm nimmt an, dass dasselbe sehr klein sei, kann aber nicht entscheiden, ob es eine Crista besitze oder nicht.

A d p. 96. 97. LINDSAY hebt die Entwickelung der Crista im Zusammenhange mit den. Sternalplatten hervor und findet ausserdem (als individuellen Befund bei 3 Embryonen von Gallus), dass dieselbe auch mit besonderem Knorpelkern, aber stets hinter und entfernt von den Anlagen der Clavicula entstehe. Damit fällt die von Görre und Horrmann behauptete Entstehung der Crista als claviculares (episternales) Gebilde. [Die angeführten Befunde und Folgerungen LindsaY's stimmen in der Hauptsache vollkommen mit meinen p. 97 ausgesprochenen Anschauungen überein].

A d p. 98. Lindsay betont ebenfalls (ohne weitere Anführung von Gründen), dass der paarigen ontogenetischen Anlage des Sternum keine phylogenetische Bedeutung zukomme, bemerkt aber später zugleich, dass die Ausbildung des Brustbeines vielleicht mit der Exspiration und der damit erfolgenden Annäherung der ventralen Rippenenden zusammenhänge. [Letzterer Ausführung vermag ich mich nicht anzuschliessen].

Ad p. 98. Anm. 3. Dollo hält unter ausführlicher Begründung seine [auch von mir Anm. 3 acceptirte] Deutung der beiden Sternalia von Iguanodon Hulke und MaRsh gegenüber fest und führt als Gewährsmänner resp. Anhänger derselben Boulenger, Gadow, Smets und Vetrer an. Hulke und Marsh haben die bezüglichen Knochen bekanntlich als Clavikeln gedeutet. Letzterer hat jedoch späterhin $(1883,1884)$ auch die Deutung als sternale Elemente für discutirbar erklärt. Doch findet er (cf. Moseley, Nature XXVIII. p. 514) selbst mit Annahme der Deutung als Sternum die Nichtexistenz der Claviculise bei Iguanodon noch nicht erwiesen, erblickt im Gegentheile an der Scapula einen Fortsatz, der auf das Vorhandensein von, wenn auch rudimentären, Schlüsselbeinen schliessen lässt. Später (Nature XXXI. p. 68) wird von ihm in der Diagnose der Iguanodontidae die Existenz einer Clavicula nicht hervorgehoben [Weiteres s. bei DoLlo]. - BAur (1885) schliesst sich ebenfalls Dollo an und homologisirt die Sternalplatten von Iguanodon den Pleurostea der Vögel, während er an der Scapula keine Andeutung findet, welche die Existenz einer Clavicula wahrscheinlich mache; bei den herbivoren Dinosauriern ist wohl wie bei Crocodilen eine Clavicula nie zur Entwickelung gelangt. Auch die Ratiten, welche zu den herbivoren Dinosauriern in nächster Beziehung stehen, besitzen nur Rudimente von Clavikeln (cf. BaUR). - VETrER betont, hauptsächlich auf die Untersuchungen von Dollo, Holke und Marsh fussend, die paarige Ausbildung des 'Sternum bei Brontosaurus, Diplodocus und Iguanodon, die unpaare bei Hypsilophodon.

Ad p. 100 und p. 137. Lindsay vermisst bei allen untersuchten Embryonen von Struthio eine Andeutung -der Crista sterni und leugnet danach direct, dass das Sternum von Struthio überhaupt einen früher bestandenen Kiel verloren habe. [Nach Ansicht einer Anzahl embryonaler Sterna von Struthio, deren Benutzung ich der Güte von Dr. GaDow verdanke, kann ich die Beobachtungen von LindsaY vollkommen bestätigen. Zugleich möchte ich hinzufügen, dass sich unter diesen Brustbeinen auch Eines (von ea. $2 \frac{1}{2} \mathrm{em}$. Länge) befand, dessen beide knorpelige Seitenplatten in der Mitte (wie es scheint - die genauere mikroskopische Untersuchung war mit Rücksicht auf die Erhaltung des Praeparates nicht ausführbar - theils hyalinknorpelig, theils fibrocartilaginös) längst verbunden waren und zwar derart, dass sie eine ventral vorspringende Längsleiste bildeten, welcher eine Längsfurche an der Innenfläche entsprach. Es fand sich somit hier eine Bildung, wohl geeignet eine Crista vorzutäuschen, doch in Wirklichkeit nicht damit identificirbar. - Den phylogenetischen Folgerungen hingegen, welche Lisosay an ihre 
ontogenetischen Befunde knüpft, vermag ich nicht ohne Weiteres zuzustimmen. Mir scheint, dass man auf Grund eines: ontogenetischen Mangels durchaus nicht immer auf eine phylogenetische Nichtexistenz schliessen darf, dass vielmehr die Vorfahren dieser oder jener Abtheilung mancherlei Gebilde besessen haben mögen, die aber so frühzeitig oder so weitgehend rückgebildet wurden, dass sie bei den jetzt lebenden Formen nicht einmal ontogenetisch mehr angelegt wurden].

Ad p. 102-112. Lindsay verdanken wir sehr interessante Mittheilungen über die Reduction der Zahl der sternalen Rippen während der ontogenetischen Fntwickelung. Die ursprünglich angelegten Rippen von Struthio vermindern sich von $6 \mathrm{zu} 5$, die von Uria von $9 \mathrm{zu} 7$, die von Sula von 8 resp. 7 zu 6 resp. 5, die von Gallus von 7 (resp. 8) zu 5 resp. 4. Die Reduction kann von vorn (am praecostalen Theile des Sternum) und von hinten (am postcostalen Bereiche) erfolgen, aber auch allein vorn (Sula). Bei Uria wurde nur noch ein kleines poststernales Skeletstückchen infolge weitgehender Reduction gefunden [cf. auch p. 108 meines Textes]. Selbstverständlich schwankt damit die Grenzbestimmung zwischen praesternalem, costosternalem und xiphosternalem Abschnitte des Brustbeines erheblich [cf. p. 112 meines Textes]. - GaDow tritt ebenfalls bei dem Vergleiche der Wirbelsäule der 3 Species von Rhea für die imitatorische Homologie der Wirbel ein [Vergl. auch Tabelle XXI. XXII, in denen ich seine Zahlenangaben noch nachträglich aufgenommen habe].

A d p. 118-128. LiNdS4Y findet anf Grund ihrer ontogenetischen Untersuchungen, dass die Trabeculae (Proc.' laterales posteriores L.) sehr früh auftretende Verlängerungen resp. Auswüchse des Sternum darstellen, deren Genese in der Hauptsache durch die Bauchmuskulatur (insbesondere den M. rectus abdominis) und auch (z. B. die intermediäre Trabecula von Larus) durch den M. pectoralis bedingt sei. Die laterale Trabecula der Galli bildet einen secundären Auswuchs der ursprünglichen intermediären. Somit wären die lateralen und intermediären Trabekeln nicht allenthalben vergleichbar. [Letztere Angabe entspricht im Wesentlichen der von mir p. 127 vertretenen Auffassung. Hinsichtlich der Beurtheilung der Natur der Trabeculae, als in der Hauptsache gesparter Skeletspangen und nicht als Auswüchse des hinteren Sternalrandes, weiche ich wesentlich von Lrndsay ab, werde aber durch die angeführten ontogenetischen Befunde nicht veranlasst, meine Auffassung aufzugeben. Dass hier in einzelnen Fällen grosse Schwierigkeiten in der Entscheidung entstehen können, habe ich bereits a. a. 0 . genügend hervorgehoben; im Übrigen aber erblicke ich in der vorliegenden Frage ein solche, bei der die vergleichend-anatomische Methode für die Erklärung die besseren Chancen als die ontogenetische verheisst]. Hinsichtlich zahlreicher Detailangaben ist auf Lindsay's Abhandlung selbst zu verweisen. - FILHOL macht specielle Angaben über das Verhalten der Trabeculae (Apophyses hyposternales F.) und erblickt in der von EYron abgebildeten Sternalform von Aptenodytes. Pennantii ein jugendliches Brustbein mit abpraeparirtem hinteren Knorpelabschnitte (incl. Trabecula). [Ich schliesse mich ihm hierin vollkommen an und ziehe damit auch meine bezüglichen Notizen (p. 126) über das Sternum des Eyton'schen Apt. Pennantii zurück]. - Das wechselnde Verhalten der Trabeculae bei den Limicolae beschreibt. ShufELIJ); bei Gallinago fand er sie einfach, bei den anderen Gattungen jederseits doppelt vorhanden. Einen noch grösseren Wechsel notirt Blasius bei den Cuculidae, wo 2 Incisuren, 1 laterale Incisur und 1 intermediäres Fenster, Übergang einer Incisur zu zweien und 1 Incisur von sehr verschiedener Tiefe zur Beobachtung kommen. Ptiloris besitzt ein oscines, Esacus ein dem von Vanellus nahe kommendes Sternum. Bei Dendrochelidon wird die Bildung der 2 grossen Fenster notirt [cf. p. 118. Anm. 2 meiner Abhandlung]. Weiteres Detail s. bei Buasius selbst. [Vergl. auch meine Tabelle XXVII, in die ich einige Notizen nach BLAsIus noch nachträglich eingefügt habe].

Ad p. 126. Anm. 1: Owen giebt die Ableitung des Sternum von Aptornis von demjenigen von Tribonyx und Ocydromus folgendermassen (Memoirs p. 340): Crista sterni bei Tribonyx gut entwickelt, bei Ocydromus recht räckgebildet, bei Aptornis beinahe ganz obsolet; Trabeculae laterales bei Tribonyx länger als das Mittelstück desSternum, divergirend, gleich hinter der letzten Rippenarticulation beginnend, bei Ocydromus verkürzt, nicht convergirend und erst in einiger Entfernung von dem Anfange des Xiphosternum anfangend, bei Aptornis Länge, Divergenz und Entfernung vom binteren Rande des Xiphosternum auf 0 reducirt. [So sehr ich geneigt bin, dieser geistreichen Vergleichung im allgemeinen Principe beizustimmen, so vermisse ich doch noch das wirklich vermittelndeGlied zwischen Ocydromus und Aptornis, um hinsichtlich der Zusammengehörigkeit beider meine Reserve völlig aufzugeben. Mir scheint, dass man bei der Ableitung des Sternum von Aptornis vielleicht nicht ganz ohne Grund auch an den Neucaledonischen Rhinochetus denken kann].

A d p. 128 f. Blasius berichtet über das sehr wechselnde Verhalten der Längen- und Breitendimensionen des. Sternum der Cuculidae, das bei Scythrops fast quadratisch, bei Eudynamus oblong, bei Centropus lang und schlunk ist.

A d p. 131 ff. Blasıus hebt die für die Cuculidae charakteristische windschiefe Krümmung des Sternum und die Vorbiegung seines binteren Randes hervor.

Ad p. 139. Anm. 1. Die Fensterbildung in der Impressio sterno-coracoidea (Fossette à la base de la face antérieure à l'apophyse hyosternale FI) von Spheniscus wird auch von FrLHou beschrieben.

Ad p. 150-152. FiLHoL macht speciellere Angaben. über das variabele Verhalten des vorderen Randes und des Apex (Sommet FI) der Crista sterni der Impennes. SHuFELDT findet die den Alcedinidae [und anderen Vögeln cf. p. 150 meiner Abhandlung] charakteristische Beziehung des vorderen Randes der Crista zu der Spina sterni 
auch bei Ceryle, erklärt dieselbe aber [abweichend von mir und, wie ieh glaube, irrthümlich] durch die Annahme eines Mangels der Spina.

Ad p. 159. Lindsay wendet sich mit Recht gegen die Vergleichung der Spina sterni mit einem Manubrium des Säugethier-Sternum.

Ad p. 164-167. Die Beziehungen des Proc. praecostalis zu Rippen werden von Lindsay ontogenetisch behandelt . und in dem auch von mir (p. 167) vertretenen Sinne entschieden. Bei Sula wird ein dreilappiger Proc. beschrieben, der aus 3 Sternocostalien hervorgehe. - Filuol macht speciellere Angaben über das Verhalten des Proc.- praecostalis (Apophyse hyosternale FI) bei den Impennes (bei Eudyptes ist er abgerundet, bei Megadyptes winkelig, bei Spheniseus sehr reducirt.

D. Secundäres Brustbein. Episternum. Ad p. 176. Houke (Adress delivered at the ann. meet of the Geol. Soc. 1883. p. 35) schliesst sich in der. Deutung des kleinen von Dollo (1882) als Episternum bezeichneten Knochens diesem Autor an; DoLLo dagegen erblickt neuerclings (1885) in demselben das Resultat der Verkalkung des Knorpels zwischen Coracoid und Sternum, vielleicht auch ein Homologon des Lophosteon.

IV. Humerus (und Schultergelenk). A d p. 194-196 un d p. 215. VETTER weist auf die verhältnissmässige Länge des Humerus von Hesperornis hin, der im Vereine mit dem Verhalten des Brustgürtels und Brustbeines auf eine frühere Flugfähigkeit schliessen und somit Hesperornis als einen extrem reducirten Carinaten auffassen lasse [vergl. auch p. 195. Anm. 1 meiner Abhandlung]. - Wredersheim notirt im Anschlusse an Marsh die alleinige Existenz des Humerus und die gänzliche resp. sehr weit vorgeschrittene Reduction der anderen Flügelknochen [cf. p. 215 meiner Abhandlung].

Ad p. 200. Bei Podasocys ist der Humerus nicht pneumatisch, bei Ceryle repraesentirt er den einzigen luftbaltigen Knochen des Flügelskeletes (SHUFELDT).

A d p. 201. 202 u n d 221. 222. Das Caput humeri bildet eine im Ganzen eiförmig erscheinencle convexe Gelenkfläche, die sich ähnlich wie bei den Crocodilen aus zwei unmerklich in einander übergehenden Abschnitten, einem cylindrischen und einem nach dem freien Ende hin verjüngten kegelförmigen zusammensetzt (AEBY).

Ad p. 204-206. Wiedersherm vermisst, Dames' Beschreibung reproducirend, bei Archaeopteryx die Crista pectoralis [vergl. dagegen meine p. 2'6. Anm. 4 gegebenen Ausführungen]. FilHor findet dieselbe (Crête pectorale Fi) bei den Impennes ansehnlich, Shufeldt ("Preaxial ridge") bei Podasocys relativ kurz.

A d p. 207-210. F几LOL beschreibt bei den Impennes einen sehr vorspringenden Processus medialis (Trochanter interne FI) und eine sehr entwickelte Fossa anconaea (Fosse sous-trochantérienne FI), die bei Megadyptes und Eudyptes einfach, bei den anderen untersuchten Gattungen durch eine besondere Leiste getheilt ist. Weiteres Detail s. in der Abbandlung selbst.

A d p. 215-218. A EBY macht kurze, aber praecise Angaben über das Ellenbogengelenk der Vögel und Reptilien [womit z. Th. die Priorität meiner bezüglichen Mittheilungen erlischt] und weist auf die verschiedenartige Umbildung und Rückbildung bei den Impennes und den Ratiten hin, wodurch Vereinfachungen der Bildung entstehen, die an gewisse Reptilien erinnern. [Ob und wie weit bei den Reptilien die Bewegung im Ellenbogengelenk auf das Handgelenk übertragen werde, wird nicht gesagt. Die einschlägige Litteratur ist (von SELENK.A abgesehen) nicht.erwähnt]. Im Übrigen ist die sehr lesenswerthe und bedeutsame Abhandlung selbst einzusehen.

Ad p. 218. 219. Der Processus supracondyloideus lateralis (Tubérosité sus-épicondylienne FI, Humeral Process ShU) ist bei den Impennes kaum angedeutet (FrLHoL), bei den Limicolae hingegen sehr entwickelt (ShUFLLIT); das Tuberculum supracondyloideum mediale (Eminence sus-épitrochléenne Fr) der Impennes bildet eine ansehnliche Vorragung (FinHoL).

Ad p. 230. Die bekannte Beziehung. der. Sehne des M; supracoracoideus (M. subclavius Su) zur Höhle des Schultergelenkes wird auch, von SuTton bei Columba angegeben und ein. Vergleich mit dem Lig. gleno-humerale der Säugethiere gezogen [womit ich nicht übereinstimmen kann].

\section{Neurologischer Abschnitt.}

Plexus brachialis. Ad p. 238 ff. FilHor giebt eine kurze Beschreibung des Plexus brachialis der Impennes (Spheniscus). Derselbe entsteht mit 4 Wurzeln vom XIII.-XVI. Spinalnerven, giebt kürzere Äste für die Mm. rhomboides und serratus profundus (von XIII.) und die Mm. serrati (von XV.), sowie für den M. supracoracoideus (XIII. + XIV.) ab und sammelt sich dann mit XIV. + XV. + XVI. zu dem Hauptstamme (Trone nerveux Fr), der sich bald in einen Rameau interne und R. externe spaltet. Ersterer giebt Rr. pectorales, den N. axillaris, Zweige für M. anconaeus humeralis und den "N. thoracique externe" ab; Letzterer zerfällt in eine Branche postérieure (N. radial), welcke den M. anconaeus versorgt und sich dann an der Streckseite des Vorderarms vertheilt und eine Branche antérieure (N. médian, musculo-cutané et cubital), welche sich bereits am Anfang des Oberarms in den.N. ulnaris und den vereinigten $N$. medianus + musculo-cutaneus theilt. [Die Beschreibung empfiehlt sich, 
was die $\mathrm{Nn}$. thoracici superiores angeht, durch Vollständigkeit, thut aber sonst zahlreicher anderer Zweige keine Erwähnung; auch statuirt sie eine sehr eigenartige Ausbildung einzelner Äste, die in vielen Stücken wesentlich von den von mir über den Plexus brachialis der Vögel erhaltenen Befunden abweicht].

A d p. 247. Cunningham, welcher früher, insbesondere Ruge gegenüber (The Relation of Nerve-supply to MuscleHomology. Journ. of Anat. and Phys. XVI. p. 1 f.), der Bedeutung der Innervation für die Bestimmung der Muskelhomologien einen gewissen, aber doch nicht zü überschätzenden Werth eingeräumt hatte, scheint sich neuerdings von der hohen Wichtigkeit derselben mehr überzeugt zu haben. Wenigstens sagt er bei Besprechung der von ibm nachgewiesenen Innervation des M. sternalis der Säugethiere 1884: "It is unnecessary here to discuss the value of nerve supply in determining muscle homology. Everyone will admit that it is without doubt the most reliable guide that we possess" ete.

\section{Myologischer Abschnitt.}

1. M. cucullaris (cucullaris + sterno-cleido-mastoideus): A d p. 302-328. Der Muskel entspricht dem Plan - profond du peaucier du cou von FuHol, sowie der tieferen Lage des Cucullaris von Gadow; beide rechnen zu demselben Systeme als oberflächliche Schichte den Sphincter colli [womit ich natürlich nicht übereinstimmen kann]. Bei Eudyptes ist der eigentliche Cucullaris nach FrLноr ein kräftiger Muskel, der vorn von dem Occipitale, dem Umkreise der Gehöröffnung, dann mit Raphe von der Dorsallinje des Halses und hinten von den Proc. spinosi der. 2 letzten Cervicalwirbel allein oder von diesen und dem 1. Dorsalwirbel entspringt und an der Clavicula und mittelst. Aponeurose an dem Insertionstheile des M. pectoralis thoracicns endet. - HeLms gielst die eingehendste Beschreibung des Muskels, dessen verschiedene Theile er, ohne ihren Zusammenhang zu berücksichtigen, einzeln unter besonderen Namen auffïhrt. [Sein Constrictor colli umfasst 2 Lagen : eine oberflächliche, die meinem Sphincter colli entspricht, und eine tiefere (Subcutaneus colli), welche den Kopftheil des Cucullaris repraesentirt; sein Dermo-furcularis stimmt in der Hauptsache mit dem Halstheile des Cucullaris, sein Dermo-spinalis mit dem Cucullaris omo-cutaneus überein; ausserdem wird noch auf den Furculo-cephalicus, der hauptsächlich den Sternocleido-hyoideus in sich enthält, hingewiesen]. Der Sphincter. ist wechselnd ausgebildet und deckt bald nur :das vordere $\frac{1}{3}$ des Cucullaris (Chauna), bald einen grösseren Theil desselben, bald nahezu seinen ganzen Halsbereich (Nothura, viele Galli, Columbae). Der von dem Kopftheile des Cucullaris abgesonderte und zu der Rückenflur in directe Eeziehung tretende Cuc. dorso-cutaneus wird bei den Limicolae (namentlich bei Scolopax) und vor Allem bei den Pici und Passeres hoch entwickelt gefunden, bei welchen beiden Familien er bis zum Os ilei reiche. [Hierbei ist der Latissimus dorso-cutaneus mit einbegriffen (den er ersetzen soll); beide sind allerdings ohne Berücksichtigung der Innervation nicht zu unterscheiden]. Vom Cucullaris propatagialis werden u. A. Andeutungen bei den Limicolae gefunden. Der $\mathrm{Halstheil} \mathrm{des} \mathrm{Cucullaris} \mathrm{steht} \mathrm{ebenfalls} \mathrm{mit} \mathrm{der} \mathrm{Rückenflur}$ im. Zusammenhange; er zeigt eine șehr wechselnde Ausbildung von einer ansehnlichen Breite (z. B. bei den Accipitres) bis zu einer recht schmalen Entfaltung (namentlich bei Pici und Passeres). Der C u c u lla ris o-mowcutane us verbindet sich insbesondere mit dem äusseren Theile der Unterflur und zeigt sich am besten bei den - Limicolae, dann auch bei den Anseres entwickelt. Weiteres zahlreiches Detail ist bei Heums selbst einzusehen. - Auf die Verbindungen des Cucullaris mit der hyosternalen Muskulatur macht auch Gadow aufinerksam.

2. M. rhomboides superficialis. Ad p. 329-343. (Cucullaris superficialis Bedpard, Trapèze Finhol, Rhomboides superficialis. Fürbringer; Rhomboides superficialis s. Spiniscapularis. GADOW). GaDow giebt einen fleischigen Ursprung an und notirt auch bei gewissen Vögeln (insbesondere Sterna, Phalacrocorax, Tantalus, minder bei den Accipitres) die Sonderung in den elavicularen und scapularen Theil; seine Insertion erstreckt sich nur auf das proximale $\frac{1}{8}-\frac{1}{2}$ (Impennes, Buceros) bis nahe zu dem Ende der Scapula (Ardea, Parra, Heliornis). Bei Eudyptes findet Fintol einen aponeurotischen Ursprung von 4 cv. und $3 \mathrm{~d}$. und eine Insertion am supracoracoidalen (acromialen Fr) Theile der Clavicula und an der Scapula; beide sind durch den Durchtritt der Gefässe gesondert. Bei Scopus inserirt der Muskel reichlich an der prox. $\frac{1}{2}$ (Beddard). Nach Gadow bei den Reptilien wohl noch mit dem Cucullaris eine einheitliche Masse bildend.

3. M. rhomboides profundus. A d p. 343-354. (C u cullaris prof and us BeDbard, Rhomboïde Finhor, Rhomboides profundus Fürbringer, Gadow). Nach Gadow meist ganz fleischig, ausserordentlich variabel. Bei Eudyptes nach Filuor breit und nicht dick von 2 ev. und 3 d. zu den distalen \& der Seapula. Bei Scopus (BedDard) an den ganzen dorsalen Rand de ${ }^{r-S c a p u l a ~ e x c l . ~ d a s ~ v o r d e r s t e ~ E n d e ~ d e r s e l b e n . ~}$

4. A. M. serratus superficialis anterior. A d p. 355-365. (Grand dentelé a n térie ur FinhoL, :Serratus superficialis anterior Fưrbrivger, P. anterior m. serrati superficialis s. thoraei-scapularis GADow). In der Regel mit 2 Zacken von den beiden ersten Rippen [resp. von cv. und 1.]"(Gadow). Bei “Eudyptes :-von, den beiden Halsrippen, mit kräftiger Sehne an den unteren Rand des verengten Theiles der Scapula (FuroL). 
4. B. M. serratus superficialis posterior. Ad p. 36j-378. (Serratus Beddard, Grand dentelé postérieur Frlhol, Serratus superficialis posterior Fürbringer, Pars posterior m. serrati superficialis s. thoraci-scapularis Gadow). Meist mit der P. anterior durch eine dünne Fascie verbunden, bei manchen Vögeln (z. B. bei vielen Raubvögeln, Khamphastus) mit derselben völlig versehmolzen (GADow; vergl. auch dessen auf p. 223 gegebene Tabelle). Bei Eudyptes (FruHou) ziemlich gross und dünn; von der 2., 3. und 4. Rippe ventral von den Proc. uncinati beginnend und an dem hinteren Winkel der Scapula inserirend; mit den Zacken des M. obliquus abdominis externus alternirend. Bei Scopus ebenfalls von der 2., 3. und -4. Rippe nach dem dorsalen Rande (Vertebral border BED.) der knappen dist. $\frac{1}{2}$ der Scapula (BEDDARD).

4. C. M. serratus superficialis metapatagialis. Ad p. 378-389. (Serratus superficialis metapatagialis Fürbringer, Pars metapatagialis m. serrati superficialis s: thoraciscapularis Gadow, Tensor cutis brachialis posterior HeLms). Nach Gadow mit 2-3, seltener 1 Zacke von den Rippen gemeinsam mit dem Serratus superficialis posterior entspringend und nach dem Metapatagium sowie den Schwungfedern 2. Ordnung gehend. Bei den Ratiten, Impennes und Tubinares vermisst. HeLMs findet einen Ursprung von 1 (Chauna, Nothura, Cypselus, Picus, vielen Passeres) bis zu 4 Rippen (Buteo), meist von 2 Rippen (bald unmittelbar unter den Proc. uncinati oder von ibren Basen selbst, bald in einiger Entfernung ventral von ihnen) und eine Anheftung an der Schulterflur und dem Metapatagium, wobei in der Regel ersterer der Hauptantheil zukommt. 'Weiteres Detail ist bei ihm selbst einzusehen.

5. M. serratus profundus. Ad p. 389-401. (A ngulaire FILHol, S.erratus profundus FürBRINGER, Gadow). Bei Eudyptes (Filhor) mit 4 Fleischzacken von ev.-2 + cv.-1 (R.), cv. (R.), 1 (R.) und 2 (R.). Gadow findet meist 3 Zacken, die von den letzten Hals- und ersten Brustrippen, oft auch vom Proc. transversus eines hinteren Halswirbels kommen können; bei Oedicnemus entspringt der Muskel nur mit 2, bei Eurystomus mit 4, hei Parra mit 4-5 Zacken.

6. M. sterno-coracoideus (superficialis et profundus). Ad p. 402-415. (Sterno-coracoidien Filhol, Sterno-eoracoideus Fürbinger, Sterno-coracoideus [costi-coracoideus und sternocoracoideus] Gadow). Bei Eudyptes vom Proc. praecostalis des Sternum nach dem distalen Bereiche der Innenfläche des Coracoid (FILHoL). Nach Gadow von clem Proc. praecostalis (Sterno-coracoideus) sowie nicht selten noch von 2 (Colymbus), 3 (Fulica), 4 (meiste Raubvögel und Psittaci) und 5 Sternocostalien (Tinnunculus, Corvjdae) (Costi-coracoideus). [Rüdoinger's Beschreibung bei Casuarius und dessen Deutung des Costo-sternalis als umgebildeter Sterno coracoideus wird reproducirt; in die Wiedergabe meiner Deutung hat sich ein Druckfehler (Innervation anstatt Insertion) eingeschlichen].

7. A. M. pectoralis thoracicus. Ad p. 415-436. (Pectoralis I. Beddard, Grand pectoral Finhol, Pectoralis thoracicus Fürbringer, Pars thoracica m. pectoralis Gadow). Bei Eudyptes von der Aussenfläche des Sternum und der Crista sterni, soweit sie nicht vom Ursprunge des M. supracoracoideus eingenommen sind, von der Clavicula und auch mit der Aponeurose des M. obliquus abdominis externus zusammenhängend. Endsehne mit dəm "Peaucier cervico dorsal" im Zusammenhange (FiLHoL). Bei Scopus nach BEDDARD von der ganzen hinteren Fläche des Sternum, der ganzen Ausdehnung der Crista sterni und dem Rande der Clavicula entspringend und an der Crista lateralis humeri inserirend, sowie auch durch Ankerung sich mit dem Tuberculum mediale verbindend. Spaltung in 2 Lagen gerade wie bei den Herodii nicht vollkommen; sondern, nur partielle Scheidung durch ein sehniges Septum. GADOw giebt zahIreiche Details, z. Th. nach den Beschreibungen früherer Autoren; das Freibleiben des Planum postpectorale vom Muskelursprunge wírd a. A. auch erwähnt. BuIx hat zahlreiche $\mathrm{W}^{r}$ ägungen der 3 Brustmuskeln (Pectoralis + Supracoracoideus + Coraco-brachialis posterior) zum Zwecke der Bestimmung der Flugcoëficienten gemacht und meist etwas grössere Zahlen als HartiNG [s. meine Anmerkungen] erhalten. Er findet das relative Gewicht dieser Muskeln zum Körpergewichte folgendermassen: Podiceps 1:10.72; mehrere Laridae 1:4.94-6.08; Anseres 1:3.9-6.2; Grus 1:5.7-5.8; mehrere Limicolae 1 : 3.34-4.55; Fulicariae 1:6.7-7.8; Columba 1:3.9-4.2; viele Accipitres 1:3.17-8.7 (mit ausserordentlichen individuellen Schwankungen); Striges $1: 6.1-10.4$; Cuculus $1: 4.92$; Caprimulgus $1: 5.39$; Cypselus $1: 4.5-4.7$; Picus 1 : 4.2-4.3; viele Passeres $1: 3.7$-5.4; die Extreme werden durch Podiceps (1:10.72) und Motacilla $(1: 3.7)$ vertreten. [Irgend welche bestimmteren Directiven mit Bezug auf Systematik, Korpergrösse oder Flugfähigkeit sind aus diesen Zịhlen nicht zu gewinnen; schon die grossen individuellen Variirungen und die Abweichungen von früheren Wïgungen beweisen genugsam, dass hier sehr versehiedenartige Ausbildungs- resp. Ernährungszustände vorliegen].

7. B. M. pectoralis propatagialis. Ad p. 437-449. (Slip for the Tensor patagii longus and brevis from the Pectoralis. I. Bepdard, Faiceau claviculairedu tensear marginal de la membrane antérieure de l'aile Fllhol (?), Pectoralis propatagialis Fürbringer, Pars propatagialis m. pectoralis Gadow). Frlhol beschreibt bei Eudyptes ein von der Clavicula kommendes Fascikel, welches vielleicht dem Pectoralis propatagialis entspricht. Bei Scopus giebt Beddard zwei Fascikel (für den Propatagialis longus und den Propat. brevis) an. Gadow. macht mehrfache An- 
:gaben über beide Fascikel, welche bald fleischig von der Clavicula entstehen, bald selnig von dem .Pèctoralis thoracicus sich ablösen. Bei den Impennes, Galli und Psittaci vermisste er die Bildung.

7. C. M. pectoralis abdominalis (Pars anterior und P. posterior). A d p. 449-462. (M. des parures Filhoc, Pectoralis abdominalis Fürbringer, Pars abdominalis m. pectoralis. Gadow, \$S ubcutaneus thoracis und Subcutaneus abdominalis. Helms). Fruhol beschreibt ihn bei Eudyptes als sehr bemerkenswerthes langes Fleischband, das von der Haut der Flanken nach der Endsehne des 11. pectoralis thoracicus geht und mit deren Aussenfläche verschmilat. Nach Gadow sind beide Theile bei den .Spheniscidae vereinigt. HeLms macht die eingehendsten Angaben über den Muskel, dessen beide Theile er mit den früheren. Autoren als verschiedene Muskeln betrachtet. Die P. posterior (Subcutaneus abdominalis Herms) geht vom Pubis aus und endet in wechselnder Weise am Seitenaste der Unterflur oder in dessen Nähe; sie ist bei den Schwimmvögeln und bei Picus. sehr breit und ansehnlich, dagegen bei Nothura und den Accipitres schwach .entwickelt. Die P. anterior (Subeutaneus thoracis HeLMs) beginnt neben oder von der Endsehne des Pectoralis thoracicus und geht direct neben oder in einiger Entfernung von dem proximalen Ende der P. posterior, an den Unterflurast resp. seine Nachbarschaft. Bei den Passeres ist der Beginn von der Pectoralis-Sehne einem grossen Wechsel unterworfen. Nothura und die Galli zeigen die stärkste Ausbildung; hier wird auch der Zusammenhang mit der Sehnenbrücke [obschon in einer von meiner Auffassung recht abweichenden Weise (Beginn von der Scapula resp. von der Sehne des "Suprascapularis" (= Scapulo-humeralis posterior mihi) beschrieben]. Weiteres Detail siehe bei HeLms selbst.

8. M. supracoracoideus. Ad p. 463-483. (Pectoralis II. Bevdard, Mo yen Pectoral FiLHoL, Su pracoracoideus Förbringer, Gadow, Subclavius Sutron). Bei Eudyptes von der Hauptfäche des Sternum, von dem Coracoid, der Membrana coraco-clavicularis und mit einigen obersten Bündeln auch von dem Ende der Clavicula und der Crista sterni entspringend und mit kräftiger, successive aus dem gefiederten Muskel hervorgehender Sehne am Anfang des Humerus inserirend. Mächtiger Muskel (FrLHor). Bei Scopus (Beddard) beginnt er von den prox. $\frac{2}{3}$ des Sternum, der dist. $\frac{1}{2}$ des Coracoid und von der Membrana coraco-clavicularis. GAvOW macht auf Grund eigener und fremder Untersuchungen zahlreiche specielle Angaben und theilt auch (auf p. 247) eine Tabelle über den sternalen Ursprung des Muskels mit, [die z. 'Th. mit meinen Befunden übereinstimmt, z. Th. von ihnen abweicht; $\mathbf{u}$. A. findet er einen auf das erste $\frac{1}{b}$ des Sternum beschränkten Ursprung bei den Tubinares, Passeres und Pici, was meinen und Forbes' Untersuchungen zưologe nur für sehr vereinzelte Formen Geltung hat]. Bei Rhinochetus entspringt der Muskel von der ganzen Länge des Sternum. "ZZugleich rechnet GADow auch Theile des M. deltoides minor ("Accessoire" und "Accessoire scapulaire" von Aurx) zu dem Muskel. In der Benennung desselben folgt er mir, hält aber auch die Deutung von AlIx (als Supraspinatus) sehr wohl vereinbar mit dem Verhalten bei den Reptilien]. Sutron beschreibt bei Columba die Einlagerung der Endsehne in das Schultergelenk. [Hinsichtlich der Deutung des Muskels erscheint ilm die von RoLiteston gegebene Vergleichung mit dem M. subelavius hinlänglich begründet (die sonstige bezügliche Litteratur wird ignorirt); ferner vergleicht er die Endsehne mit dem Lig. gleno-humerale der Säugethiere, welcher Auffassung ich durchaus nicht folgen kann].

9. M. coraco-brachialis externus s. anterior. Ad p. 483-494. (Internal d el toid BeDdari, Coraco-brachialis anterior s. externus FÜrbringer, Coraco-brachialis anterior Gadow). Bei Scopus von dem Acrocoracoid zur ventralen Fläche der Crista lateralis humeri, vor und z. Th. unter der Insertion des Pectoralis thoracicus (BEDDaro). Nach GaDow vom vorderen Vorsprunge des Coracoid [= Acrocoracoid] nach der Aussenfläche der oberen Armbeinleiste. Weitere Details s. in seiner Abhandinng.

10. M. coraco-brachialis internus s. posterior. Ad p. 494-506. (Coraco-brachialis exteinus Bedpard, Coraco-brachial Filhol, Coraco-brachialis posterior s. internus Fürbringer, Coraco-brachialis posterior Gabow). Bei Eudyptes ein sehr kräftiger mit dem Supracoracoideus verbundener Muskel, der vom dist. Theil der Aussenfläche des Coracoid nach dem Rande der Fossa anconaea geht, wo er sehnig neben dem Subscapularis inserirt (FILHOT). Bei Scopus von dem sternalen Ende des Coracoid (BEDDARD). GaDow giebt eine eingehende Beschreibung und betont ansser dem coracoidalen auch den sternalen Ursprung; nur vom Coracoid komme der Muskel bei Struthio, Otis, Fulica, den Galli, Columbae und den untersuchten Passeres [was z. Th. mit meinen Befunden nicht ganz übereinstimmt]. Im Übrigen wird der Coraco-brachialis pusterior von ihm mit dem zweiten Coraco-brachialis der Säugethiere, insbesondere der Monotremen verglichen.

11. A. M. biceps brachii. Ad p. 506-j21. (Biceps Bevdird und FllHol, Biceps bräehi Fürbringer, (Gadow). Bei Eudyptes fehlend (Frlhol, Gadow). Bei Scopus mit doppeltem Ursprunge und mit doppelter Insertion an Radius und Ulna (BEDDard). Nach Gadow bei den Ratiten nur vom Coracoid entspringend und am Radius inserirend. [Ich' fand bei Struthio, Rhea und Casuarius auch eine deutliche ulnare Insertion]. Weiteres Detail s. bei Gadow.

11. B. M. biceps propatagialis. Ad p. 521-530. (Biceps slip to the Patagium. BEDDArD, Biceps propatagialis Fürbringer, Pars propatagialis des M. biceps Gadow). Bei Seopus fehlend (BEDdaRd). Gadow reproducirt die von Garkod über diesen Muskel mitgetheilte Liste. ... ... $\ldots$ 
12. M. brachialis inferior. Ad p. 530-532. (Brachialis internus Beddard, Brachial antérieur Frlmol, Brachialis inferior Fürbringer, Gadow). Bei Eudyptes nach FunHol fehlend, nach GaDow dagegen besonders stark entwickelt und an Radius und Ulna inserirend. Bei Scopus (BEDDARD) breit und flach von der tiefen Fossa am Ende des Humerus nach dem Anfange der Olna.

13. A. M. latissimus dorsi anterior. Ad p. 533-545. (Anterior portion of the Latissimus dorsi Bedpard, Faisceau spinal du Grand dorsal FrlHol, Latissimus dorsianterior Fürbinger, Vorderer Theil des Latisimus dorsi Gadow). Bei Eudyptes ein sehr entwickelter Muskel, der von den Proc. spinosi der 3 letzten Cervical- und der 3 ersten Dorsalwirbel entspringt und nach dem Humerus unterbalb der Fossa anconaea geht, wobei seine.Sehne zusammen mit der des Latissimus dorsi posterior durch einen am Collum scapulae befestigten Faserring verläuft. Dieser Faserring (Schlinge), dessen auch GaDow Erwähnung thut und der nach FILHOL mit dem Deltoides major zusammenhängt, wird von FILHOL zur Scapula. gerechnet, von SutTon als ein Product der degenerativen Metamorphose von Fasern des M. deltoides betrachtet. [womit ich nicht übereinstimme]. BEDDARD beschreibt $u$. A. bei Scopus auch den Zusammenhang mit der humeralen Ankerung des M. anconaeus scapularis (Accessory tendon of Anconaeus longus). Bezüglich GADow's Beschreibung ist die Abhandlung selbst einzusehen.

13. B. M. latissimus dorsi posterior. Ad p. 546-563. (Posterior half of the Latissimus dorsi Beppard, Faisceau dorsal du Grand dorsal FilHol, Latissimus dorsi posterior Fürbringer, Hinterer Theil des Latissimus dorsi Gadow). Bei Eudyptes von der Crista ilei und der Praesacralrippe mit sehnigem Ursprunge und sehniger Insertion, mit Latissimus dorsi anterior durch die Faserschlinge tretend und ibn dabei kreuzend. Kleiner als Ersterer (FrLHoL). Bei Scopus breiter als Latissimus dorsi anterior, proximal von ihm und mit der humeralen Ankerung des Anconaeus scapularis ebenfalls verbunden zum Humerus (BEDDARD). GaDow giebt eine umfassende Darstellung und legt besonderen Nachdruck auf die Latissimus dorsi anterior und posterior verbindende Fascie, die aber oft äusserst dünn ist (Näheres s. seine Beschreibung p. 227). Bei den Impennes, den Sumpfrögeln und bei Cypselus sind beide Mm. latissimi dorsi (anterior und posterior) stark entwickelt, bei den meisten Tauben ist der Latissimus posterior schwach und kann hier selbst bei einzelnen (Columba livia, C. oenas, Phaps) fehlen; HAswell's Angaben hinsichtlich des Mangels bei vielen Columbae werden zurückgewiesen.

13. C. M. latissimus dorsi metapatagialis und dorso-cutaneus. Ad p. 563-576. (Tenseur de la membrane axillaire Frhor, Latissimus dorsi metapatagialis und Lat. d. dorsocutaneus Fürbringer, Pars metapatagialis des M. latissimus dorsi Gadow, Dermoiliacus Hexms 1]). Bei Eudyptes von den Proc. spinosi des 3., 4. und 5. Dorsalwirbels mit vorderen und mittleren Fasern zu der Sohulterflur und dem Metapatagium, mit hinteren zur Aponeurose des Obliquus abdominis externus (Frlfor). Nach Gadow ein undeutliches, von dem hinteren Bauche des Latissimus dorsi sich ablösendes Bündel, das sich sehnig am Banche des M. anconaeus longus anhefte. Scheint nach ihm bei den Ratiten, den. Spheniscidae, Colymbus, Grus leucogeranus, den Psittaci und Cypselus zu fehlen [was z. Th. mit meinen Befunden übereinkommt, z. Th. davon abweicht]. HeLms macht zahlreiche Detailangaben über den Muskel, der bald vom oberen oder vorderen Rande des Os ilei (Anas clangula, Limicolae, gewisse Raubvögel), bald von den Proc. spinosi eines, seltener zweier hinterer Dorsalwirbel (Colymbus, mẹiste untersuchte Anseres, Chauna, Nothura, Galli, Columbae, gewisse Raubrögel) beginnt und meist nach der Schulterflur und dem Metapatagium (Colymbus, Anseres, Chauna, Columba, Accipitres, Striges) oder zur Schulterflur und Spinalflur resp. Mittelrücken (Limicolae, Nothura, Galli, Goura) verläuft. [Die zur Schulterflur und zu dem Metapatagium sich begebenden Fasern entsprechen dem Latis-simus dorsi metapatagialis, die nach der Spinalflur gehenden dem Latissimus dorsi dorso-cutaneus; ausserdem gehört hierher noch der Rückenabschnitt des bei den Passeres beschriebenen dorsalen Muskelzuges, den HeLms zu dem. Dermo-spinalis rechnet $\left.{ }^{\mathbf{1}}\right)$ ].

14. A. M. deltoides propatagialis (incl. Propatagialis longus et brevis). Ad p. $576-614$. (Tens or patagii lóngus et brevis BeDdand, Tenseur marginal de la membrane antérieure de l'aile. Funhol, Deltoides propatagialis (longus und brevis) Fürbringer, M. propatagialis lóngus. und brevis Gadow). Bei Eudyptes z. Th. atrophisch oder wegen Abwesenheit des Propatagium verändert, sehr nahe an dem Rande des Humerus und Radius verlanfend, hier befestigt und zum Metacarpus gehend (FILHOL). Bei Scopus einheitlich von Clavicula und Scapula entspringend und in einen Muskel übergehend, der sich im distalen Bereiche in 2 Theile spaltet, welche in die Sehnen des Propatagialis longus und brevis sich fortsetzen. Ersterer geht zum Carpale radiale, giebt aber in der Fllenbeuge eine schmale, doch nicht schwache Ankerung $[=\sigma]$ an den.

1) Auch der hintere Abschnitt des Dermo-spinalis Helns bei den Pici und Passeres gehört hierher (cf. p. 819). Den Humero-spinalis HeLMs, der bei Picus zwischen Schulterflur und Rückenflur verläuft, vermag ich nirgends mit Sicherheit unterzubringen; jedenfalls wird ein Theil des Cucullaris oder des Latissimus dorsi, wenn nicht ein glatter Bautmuskel darunter verstanden. 
Propat. brevis ab. Letzterer hängt durch Elastik mit der Crista lateralis humeri zusammen und spaltet sich in einen proximalen (Outer tendon $=\gamma$ ) und distalen Sehnenzug (Inner tendon $=a+\beta$ ), von denen der proximale zur Vorderarmfascie (Radial aponeurosis), der distale nachdem er sich mit Pectoralis propatagialis verbunden hat, mit 2 Zipfeln zur Selne des Extensor metazarpi radialis geht und anch mit $\sigma$ zusammenhängt (BEDDARD). GADOW macht zahlreiche Detailangaben, worunter namentlich die GARroD'schen Befunde bei den Coccygomorphae und Aegithognathae ihrer Wichtigkeit entsprechend ausführlich behandelt werden. Abgesehen von den Ratiten wird auch bei den Impennes Nichtexistenz des Propatagialis brevis angegeben, während für den Propat. longus bei allen Vögeln ein mehr constantes Vorkommen statuirt wird. [Wenn ich Gadow recht verstehe, fasst er die Sehne (Propatagialis) zusammen mit dem ganzen Complexe der hier in Frage kommenden Gebilde als einen mehrköpfigen (in maximo 4köpfigen) Muskel auf, dessen Köpfe durch die Mm. deltoides, pectoralis, biceps und cucullaris propatagialis repraesentirt werden, und bezeichnet denselben als M. propatagialis. Das kommt im Ungefähren mit meiner Auffassung überein, deckt sich aber nicht vollkommen mit ihr. Weiterhin wird die nach meiner Ansicht höchste Specialisirung des Propat. brevis bei den Pici und Passeres als einfachstes Verhalten bezeichnet, eine Anschauung, die allerdings Recht hat, wenn die specialisirte und damit vereinfachte Bildung an sich, aber ohne Beziehung auf ihre Genese (Phylogenese) betrachtet wird].

14. B. M. deltoides major. Ad p. 614-634. (Deltoid BEDdard, Deltoïde postérieur Filhor, Deltoides major Fürbringer, Gadow). Bei. Eudyptes mit 2 Sehnen vom Acromion und dem acromialen Theile der Clavicula entspringend und an dem prox. Abschnitte der Aussenfläche des Humerus inserirend. Zugleich mit der Faserschlinge für die $\mathrm{Mm}$. latissimi dorsi zusammenhängend (FruHoL). Bei Scopus beginnt er vorn fleischig und hinten kurzsehnig von der Scapula neben der Verbindung mit dem Coracoid und geht an die Aussenfläche der Crista lateralis humeri (BeDdard). GADOw theilt zahlreiche Details mit. Der Muskel kommt in wechselnder Weise: I. von der Gelenkkapsel des Schultergelenkes und dem Os humero-capsulare und II. von dem Acromion, dem dorsalen Ende der Clavicula und hänfig auch vom Proc. procoracoideus (Innenvorsprung des vorderen Endes des Coracoid $G_{A}$ ); bei Gallus beginnt er hauptsächlich von dem Lig. acrocoraco-acromiale. Am stärksten ist er bei den Accipitres, am schwächsten bei den Impennes und Psittaci [Meine Befunde weichen z. Th. von den von GaDow mitgetheilten ab].

14. C. M. deltoides minor (Pars dorsalis und P. ventralis). Ad p. 634-649. (A c cessoire coraeoidiendu moyen pectoral [ $=$ P. ventralis] Funol, Deltoides (Teres) minor Fürbringer, Deltoideus minor (GaDow 1]). Bei Eudyptes beginnt der ventrale Theil vom vord. Rande des Sternum und von der Membrana coraco-clavicularis, geht, bedeckt vom M. supracoracoideus, nach vorn und schlingt sich um das Coracoid herum, um am Tuberculum laterale zu inseriren. Der dorsale Theil repraesentirt ein unbedeutendes Bündel, das von der Innenfläche des Acrocoracoid nach dem Humerus geht. Ein Theil der Sehne verschmilzt mit dem Deltoides propatagialis (FILHOL). Nach GaDow beginnt die Pars dorsalis [resp. der ganze Muskel bei mässiger Entwickelung] vom Anfang der Scapula und dem dorsalen Ende der Clavicula und inserirt zwischen den beiden Theilen (I und II) des Deltoides major am Humerus. [Hinsichtlich der P. ventralis siehe Supracoracoideus].

15. A. M. seapulo-humeralis anterior. Ad p. 649-660. (S capulo-humeralis anterior FürBRINGER, Seapuli-humeralis anterior Gabow). Von der Aussenflache des basalen $\frac{1}{3}$ der Scapula - Tuberculum mediale humeri, etwas nach aussen und hinten von der Fossa pneumo-anconaea. Bei den Spheniseidae, Leptoptilus, Upupa und einigen Passeres ganz stark, bei Casuarius, Apteryx, den Galli und Accipitres recht schwach, bei Struthio (und Otis) fehlend (GARrod). [Die Angaben, die Ratiten und Impennes betreffend, beziehen sich vermuthlich auf einen anderen Muskel].

15. B. M. scapulo-humeralis posterior. Ad p. 660-670. (Teres BEDdard, Grand rond Frlhor, Scapulo-humeralis posterior FörbRINGer, Scapuli-humeralis posterior Gadow). Bei Eudyptes (FinHor) ein grosser und dicker Muskel, der von den dist. $\frac{2}{3}$ der Aussenflüche der Scapula mit kräftiger Sehne nach dem Tuberculum mediale humeri geht; oberflächlich aponeurotisch. Nach Bedpard bei Scopus nahezu vom ganzen ventralen Rande der Scapula [Aussenfläche vermuthlich aus Versehen nicht erwähnt] zum Humerus nehen der Fossu pneumo-anconaea, direct vor dem Ursprunge des Anconaeus humeralis. Auch durch ein kleines Sehnenbündel mit dem Anconaeus scapularis in Verband stehend [= scapulare Ankerung dieses Muskels]. Gadow macht zahlreiche Detailangaben. Vom dist. $\frac{1}{2}$ (Ciconia) bis $\frac{3}{4}$ (Spheniscidae) der Scapula nach dem Humerus, distal neben der' Innenfläche des Tuberculum mediale. Bei Struthio-Embryonen nur von den mittleren 3 der Scapula, bei Dromaeus vielleicht fehlend. Nach GADow dem gleichnamigen Muskel der Saurier und Amphibien vergleichbar [mit welcher letzteren Homologisirung ich nicht ganz übereinstimme]. .

1) Diese Bezeichnung wird von GADOW für den ganzen Muskel bei geringerer oder mittlerer Ausbildung gebraucht, dagegen bei höherer Ausbildung nur für die P. dorsalis angewendet, während dann die P. ventralis in der Hauptsache zu dem Supracoracoideus gerechnet wird (vergl. diesen). 
16. M. subcoracoscapularis (A. Caput coracoideum, B. Cap. scapulare internum, C. Cap. scapulare externum). Ad p. 670-689. (Coraco-brachialis [= A.] und Subscapularis $[\doteq B$. und C] Bedpard; A c cessoire coracoudien d u sous-scapulaire [=A.], Sous-scapulaire [= B.] und Petit rond [=C.] Frthol; Pars coracoidea, P. scapularis interna und P. scapularis externa m. subcoracoscapularis Fürbringer; Subcoracoideus [=A.] und Subscapularis (internus und externus) [= B. und C.] Gavow). Bei Eudyptes nach FuLHoL: A. von der Membrana coraco-clavicularis, dem Proc. medialis des Coracoid und dem Anfange des Sternum, B. von der Innenfläche des geraden Theiles der Scapula, C. kräftig von der Aussenfläche der Scapula vor dem Ursprun : des Scapulo-humeralis posterior, den Serratus superf. anterior deckend. Alle drei inseriren verbunden am Tuberculum mediale humeri. Bei Scopus bietet der Muskel keine Besonderheiten dar (Beddard). Gadow theilt zahlreiche Specialitäten, zumeist nach den Untersuchungen früberer Autoren mit und weisst auf die betrïchtlichen Variirungen der Grösse der einzelnen Köpfe (Theile) hin. A. notirt er bei den Galli und Columbae als relativ am kräftigsten, vermisst ihn dagegen [in Differenz mit meinen und anderer Autoren Angaben] bei Struthio; B. erstreckt sich über die prox. $\frac{1}{2}$ bis $\frac{2}{8}$ der scapularen Innenflüche, G. über das prox. $\frac{1}{5}$ (Spheniscidae, Galli) bis $\frac{1}{3}$ (Anseres etc., Ardea, Tinnunculus) des ventralen Randes und angrenzenden Aussen- und Innensaumes der Scapula. In der Deutung des Muskels wird auch die Zulässigkeit der SeLenKA'schen Vergleichung mit dem Coraco-brachialis offen gehalten [worin ich anderer Ansicht bin].

17. A. M. anconaeus scapularis. Ad p. 690-708. (Triceps Iongus BEDdARD, Longue portion da triceps brachial Frrnol, Caput scapulare m. anconaei (Anconaeus scapularis) Fürbringer, Langer Theil des Triceps eubiti s. Anconaeus (Scapuii-cubitalis) Gavow). Fuдно beschreibt bei Eudyptes die beiden Köpfe der Impennes, von denen der claviculare von der hinteren Fläche des acromialen ${ }^{*}$ heiles der Clavicula, der scapulare vom axillaren Rande der Scapula beginnt. Beide vereinigen sich mit einander und später mit dem Anconaeus humeralis, gehen in eine Sehne über, welche die laterale Ulnarpatelle (Sesamoïde externe du coude) einschliesst. und enden an dem Tuberculum superius des Olekranon. Nach BEddakn bei Scopus zar Hälfte sehnig von der Scapula neben dem Schultergelenke beginnend, weiterhin auch eine scapulare und humerale Ankerung aufnehmend (erstere beginne vom Scapulo-humeralis posterior) und lrräftig am vorderen Rande des Olekranon inserirend. GADow giebt dem Muskelkopfe eine relativ grosse Selbständigkeit; die humerale Ankerung findet sich besonders bei den Raubvögeln.

17. B. M. anconaeus coracoideus. (Tendom. anconaei coracoidei und Expansor secundariorum). Ad p. 708-721. (Expansor secundariorum Bendard und Gadow, Caput coracoideum m. anconaei (A ncónaeus coracoideus) Fürbringer, Theil des M. metapatagialis Gadow 1)). Bei Scopus scheinbar fehlend (BEddand). Gadow reproducirt in der Hauptsache Garrod's und meine Mittheilungen über das betreflende Gebilde.

17. C. M. anconaeus humeralis. Ad p. 721-734. (Triceps BEDdARd, Vaste externe et interne Frrhor, Caput humerale m. anconaei (Anconaeus humeralis) Fürbringer, Kürzerer Theil des Triceps cubiti s. anconaeus [Homero-eubitalis] Gadow). Bei Eudyptes nach Filног mit äusserem und innerem Kopfe, ron denen der erstere sich dem Anconaeus scapularis näher anschliesst, während der letztere in eine lange und schlanke Sehne übergeht, welche das "Sesamoïde interne du coude" enthält. Bei Scopus in der gewöhnlichen Weise von der Dorsalfläche des Humerus, weiterhin mit Anconaeus scapularis verbunden (BEDDARD). GADOW giebt eine eingehende Beschreibung und macht besonders auf den distalen accessorischen Kopf (Anconaeus IV. s. brevissimus der Autoren) aufmerksam.

1) GADow lässt den M. metapatagialis aus der Pars metapatagialis m. serrati, der P. metapatagialis m. latissimi dorsi und aus dem M. expansor secundariorum zusammengesetzt sein. [Diese Gruppirung entspricht nur z. Th. meiner Auffassung des Metapatagialis (ef. p. 735)]. 